\title{
Jet Energy Scale and Resolution in the High-Granularity Timing Detector in ATLAS Upgrades at HL-LHC
}

\author{
Asmaa Aboulhorma and Farida Fassi \\ Mohammed V University in Rabat, Morocco
}

\begin{abstract}
The large increase of pileup is one of the main experimental challenges for the High Luminosity-Large Hadron Collider (HL-LHC) physics program. HL-LHC is expected to start in 2027 and to provide an integrated luminosity of $3000 \mathrm{fb} 1$ in ten years, a factor 10 more than what will be collected by 2023 . A powerful new way to address this challenge is to exploit the time spread of the interactions to distinguish between collisions occurring very close in space but well separated in time. A High-Granularity Timing Detector (HGTD), based on low gain avalanche detector technology, is proposed for the ATLAS Phase-II upgrade. Covering the pseudo rapidity region between 2.4 and 4.0, with a timing resolution of 30 ps for minimum-ionizing particles, this device will significantly improve the performance in the forward region. The improvement of the jet energy scale and resolution in the forward region by reducing the pileup track contamination in hard scatter jets from nearby pileup interactions is presented. The impact of HGTD in reducing pileup track contamination in the jets reconstruction in the forward region is investigated. The performance is evaluated in terms of jet energy response and resolution as a function of pseudo rapidity $\eta$, transverse momentum $p_{T}$.
\end{abstract}

Keywords: HL-LHC, ATLAS, HGTD, jet energy scale, jet energy resolution, jet- $p_{T}$ correction DOI: $10.31526 /$ ACP.BSM-2021.5

\section{INTRODUCTION}

The high-luminosity phase of the Large Hadron Collider (HL-LHC) is Scheduled to begin in 2027 and will deliver associate degree integrated luminosity of up to $4000 \mathrm{fb}^{1}$ in concerning 10 years. The instant luminosity ought to reach up to $7.5 \times 10^{34} \mathrm{~cm}^{2} \mathrm{~s}^{1}$, compared to the present value of $210^{34} \mathrm{~cm}^{2} \mathrm{~s}^{1}$. associate upgrade of the ATLAS detector [? ] are required before the beginning of this new phase to deal with the high-radiation environment and therefore the large increase within the number of collisions per bunch crossing.

In the new HL-LHC conditions, pileup will be one of the biggest challenges. For the nominal operating scheme, the interaction region will have a Gaussian spread of $45 \mathrm{~mm}$ along beam axis 1 and a pileup of 200 simultaneous pp interactions on average $(<\mu>=200)$, corresponding to an average interaction density of 1.8 collisions $/ \mathrm{mm}$, as shown in Figure 1 (left), compared to the 0.24 collisions $/ \mathrm{mm}$ of run 2 .
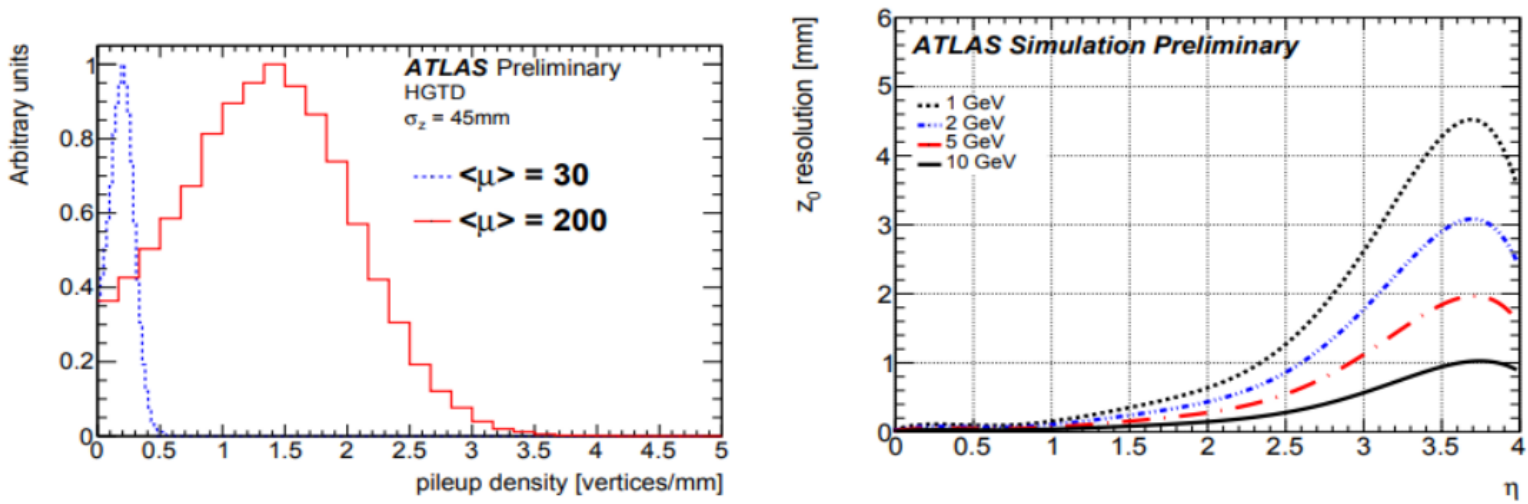

FIGURE 1: Left: Current and HL-LHC local pileup vertex density. Right: The resolution of the longitudinal track impact parameter, $Z_{0}$, as a function of $\eta$ for different $p_{T}$ values

Under these conditions, a major challenge is to reject the charged particles generated by the pile-up. The resolution of the longitudinal trajectory impact parameter $\left(Z_{0}\right)$ must be better than the average distance between interaction points $(0.6 \mathrm{~mm}$ for HL-LHC). The $Z_{0}$ resolution is well below this limit in the central region, but becomes very large in the forward region, reaching 
up to $5 \mathrm{~mm}$ for particles with low transverse momentum $\left(p_{T}\right)$ as seen in Fig. 1 (Right). As a result, tracks can not be associated to the correct vertices in an unambiguous way, leading to reduced performance in terms of heavy flavour tagging, lepton isolation and the identification of jets originating from pileup interactions

\section{DETECTOR}

A powerful new way to address the challenging pileup conditions of HL-LHC is by imploying the time spread of the different interactions during a single beam crossing. Figure 2 illustrates the time spread with an RMS of 180 ps. The position and time of the interaction points during a single cluster crossing are shown. Dotted lines represent the positions of the reconstructed vertices in the ITk. Since the time and position of the interaction are uncorrelated, if it is possible to measure the time of the traces from a vertex with enough accuracy, it's miles feasible to determine whether they originate from different interactions or a single interaction.

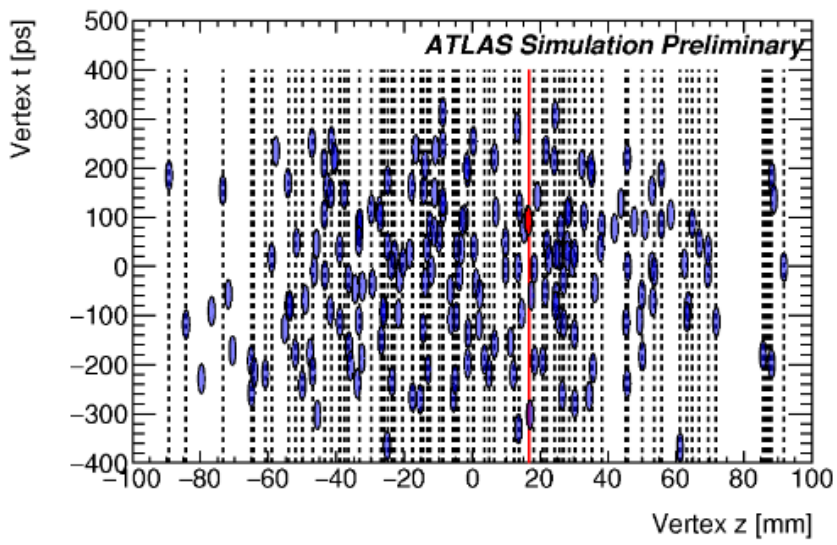

FIGURE 2: Position and time of the interaction points during a single bunch crossing $<\mu>=200$ [?]

In Figure 2 the red point represent the signal interaction, at the same position there are also other pileup interactions, but the timing information separates these interactions.

In this context, the High Granularity Time Detector (HGTD) is introduced [? ? ], to augment the new all-silicon Iner Tracker in the forward region. Offering a time resolution of $30 \mathrm{ps}$ in the forward region, this detector will be able to reject stacks from $|\eta|=2.4$ up to $|\eta|=4$. More generally, this detector will improve the performance of the ATLAS detector in the forward region to a level similar to that of the central region and will provide a powerful tool for luminosity measurements.

The HGTD consists of two circular disks placed in between the ID and the end cap of the calorimeter at $z= \pm 3.5 \mathrm{~m}$ with an active area at $120 \mathrm{~mm}<R<640 \mathrm{~mm}$, corresponding to $2.4<|\eta|<4$ as shown in Figure 3. The space allocated to the HGTD for ATLAS HL-LHC upgrade is quite limited, which defines some of the HGTD geometrical parameters, its thickness should not exceed $125 \mathrm{~mm}$ including $50 \mathrm{~mm}$ of moderator to protect the ITk and the HGTD from back-scattered neutrons. In order to ensure a timing resolution of 30 ps per track over all the HL-LHC, two layers of active sensors will be built with an additional effective layer at low radius. This additional effective layer will be achieved by increasing the overlap of the modules for $R<320 \mathrm{~mm}$ from $20 \%$ to $80 \%$.

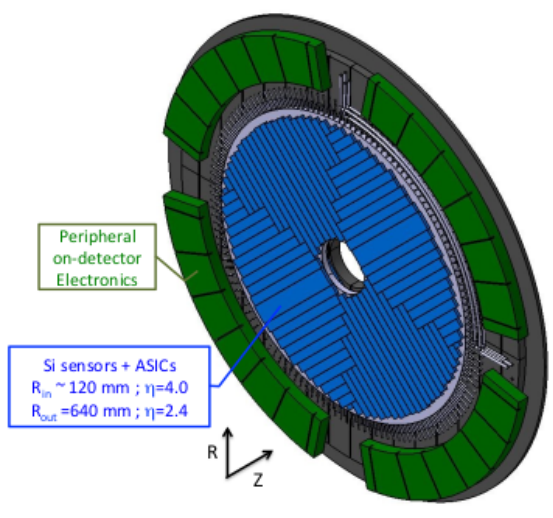

FIGURE 3: Illustration of the HGTD.The blue represent the active region and the green the off detector electronic 


\section{PERFORMANCE}

The precision time measurement capability of the HGTD can be used to enhance considerably the reconstruction of different physics objects. This can be accomplished by rejecting out-of-time pileup that could contaminate objects thus reducing their reconstruction efficiency. In order to study the performance of the HGTD and to optimize the detector layout, events [? ] of jets, have been fully simulated.

\subsection{Suppression of pileup jets}

Pileup is very crucial for the reconstruction and identification of objects at the HL-LHC. In the case of jets, pileup can affect the reconstruction in a different ways. One is that particles from a pileup interaction can infect a jet from hard scatter and change its reconstructed energy. modify its reconstructed energy. Pure pileup jets from an interaction other than hard scatter can also be created, due to a QCD process during the pileup interaction, more likely at high $p_{T}$, or because of a random combination of particles from different interactions, more likely at low $p_{T}$.
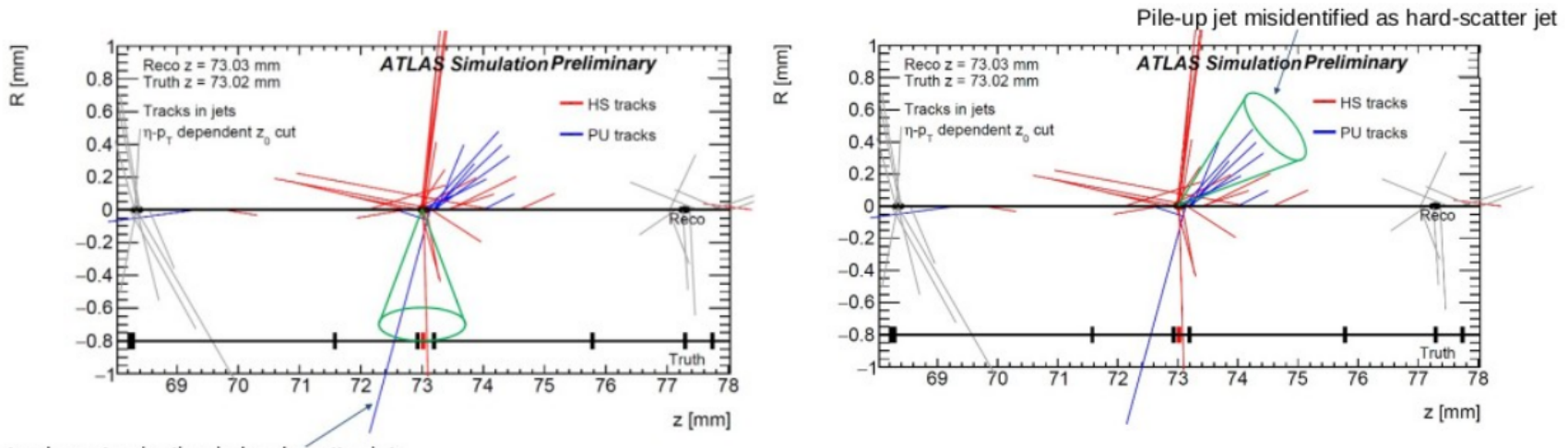

Pile-up track contamination in hard-scatter jets

FIGURE 4: All tracks associated with primary vertices illustrating the PU contamination

A key element for the rejection of pileup jets is the association of the jets tracks with the primary vertices. The main variable used to separate the pileup vertices from the hard scatter one is $R_{p_{T}}$, the ratio between the sums of the $p_{T}$ of all tracks originating from the hard scatter vertex associated with the jet and the $p_{T}$ of the jet. This variable should be large for the hard scatter jets and low for pileup ones. Unfortunately in high pileup environment and especially in the forward region it is possible to have pileup vertices that appear to be merged with the hard scatter vertex. In that case the discriminating power of this variable is greatly reduce.

\subsection{Jet Energy Response and Resolution}

Precise knowledge of the jet energy resolution is important for detailed measurements of SM jet production, measurements and studies of the properties of the SM particles that decay to jets, as well as searches for physics beyond the SM involving jets in ATLAS detector, especially for the HGTD.

Jet Energy Response (JER) is defined as the ratio of calorimeter jet $\left(p_{T_{\text {reco }}}\right)$ to the truth-jet $\left(p_{T_{\text {truth }}}\right)$ energies.

The mean of the estimated JER is determined using the Gaussian fit of the the distribution $p_{T_{\text {reco }}} / p_{T_{\text {truth }}}$, where $p_{T_{\text {reco }}}$ stand for the transverse momentum of the reconstructed calorimeter jet and $p_{T_{\text {truth }}}$ is the transverse momentum of the jet reconstructed from all the generated stable particles (excluding neutrinos)[?].
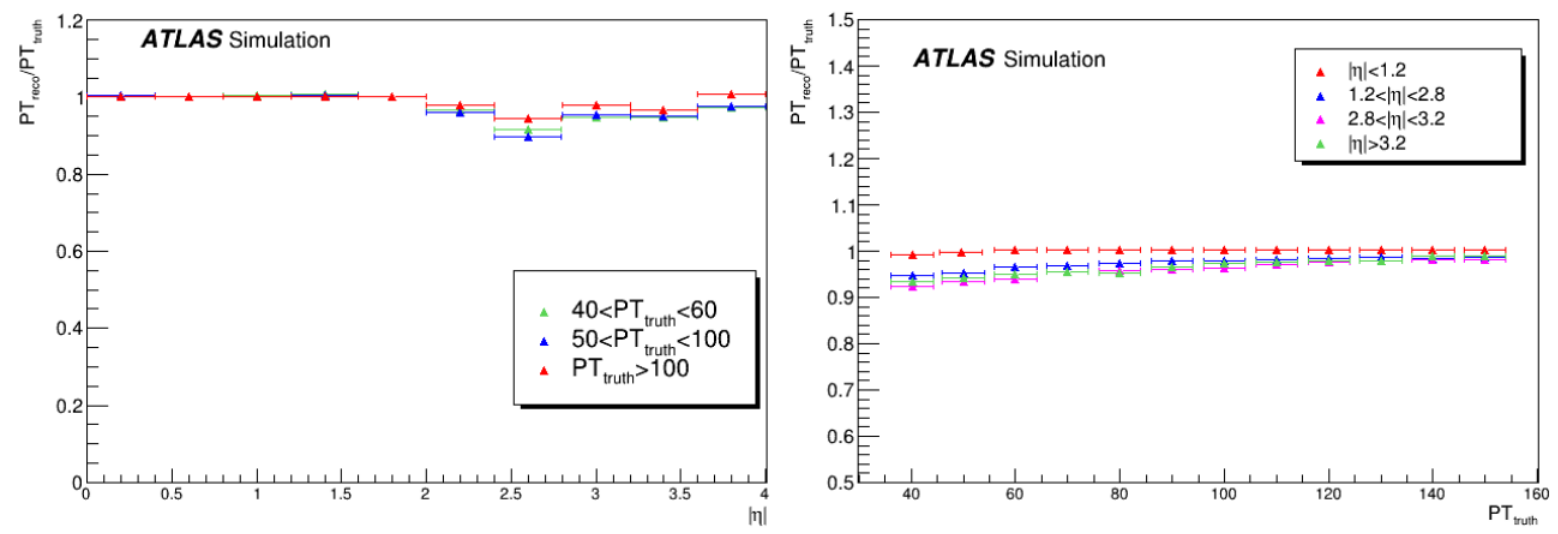

FIGURE 5: JER as a function of $\eta\left(\right.$ left) and as a function of $p_{T_{\text {truth }}}$ (right) for raw calorimeter jets 
Jet Energy Resolution is essentially a measure of the detector's ability to resolve the difference in energy between two jets of similar total energy. The jet energy resolution is parameterize as follows:

$$
\frac{\sigma\left(p_{T_{\text {reco }}} / p_{T_{\text {truth }}}\right)}{\mu\left(p_{T_{\text {reco }}} / p_{T_{\text {truth }}}\right)}
$$

where $\sigma$ and $\mu$ are evaluated from the Gaussian fit of the distribution $p_{\text {Treco }} / p_{T \text { truth }}[?]$.
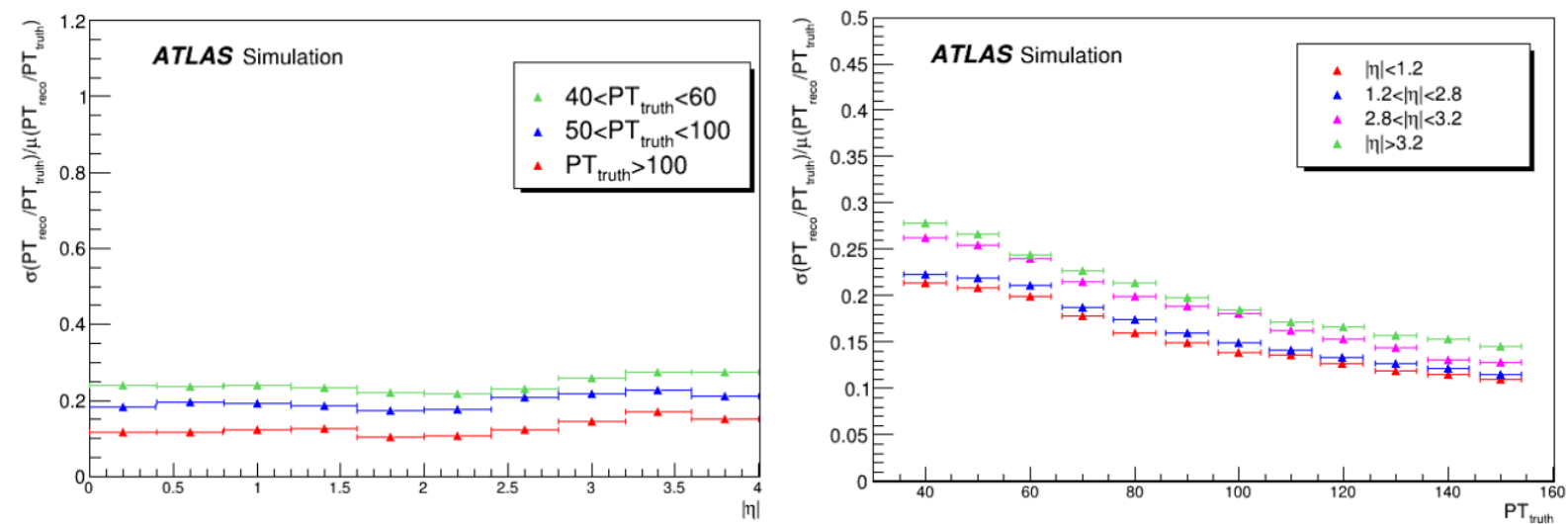

FIGURE 6: The transverse jet energy resolution as a function of $\eta$ (left) and as a function of $p_{T_{\text {truth }}}$ (right) for raw calorimeter jets

The comparison of the jet resolution for the various eta and pt bins is shown in Figure 6. A good resolution is seen in the central region of the detector, however it is reduced in the forward region for low $p_{T}$ regime. This is due to the Pileup-Track contamination at the hard scatter jets as illustrated in Figure 4 (left).

\subsection{Jet $p_{T}$ correction}

The purpose of the Jet $p_{T}$ correction is to remove the Pileup Track energy, from the hard scatter jet energy deposited in the detector and eventually reconstructed. The correction method consists in assigning tracks to primary vertices-a track is mapped to a vertex if its origin is geometrically compatible in the longitudinal trajectory impact parameter $Z_{v t x}$ with the vertex position. This compatibility can be determined by the resolution on the track $Z_{0}$ impact parameter as follows:

$$
\frac{Z_{0}-Z_{v t x}}{\sigma}<2
$$

The Jet $p_{T}$ is improved by removing the E/P of this PU-track as follows:

$$
p_{T_{J e t-c o r r}}=p_{T_{J e t}}-\sum E / P * p_{T-P U T r a c k}
$$
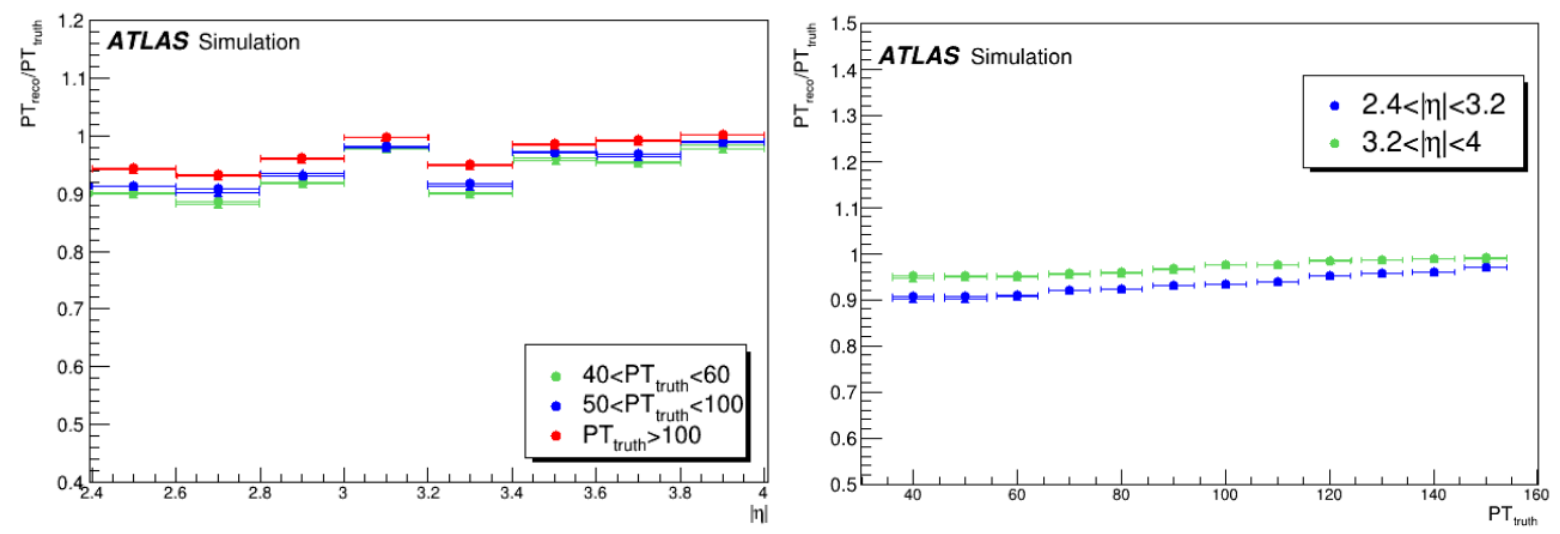

FIGURE 7: JER as a function of $\eta($ left $)$ and as a function of $p_{T \text { truth }}$ (right) before and after correction

Figure 8 (right-hand) shows that the jet response remains unchanged, after applying the correction for different eta bins. The jet resolution has been improved by $3.5 \%$ and $1.78 \%$ for $40<p_{T}<60$ and $50<p_{T}<100$, respectively, as illustrated in Figure 8 (left-hand). 

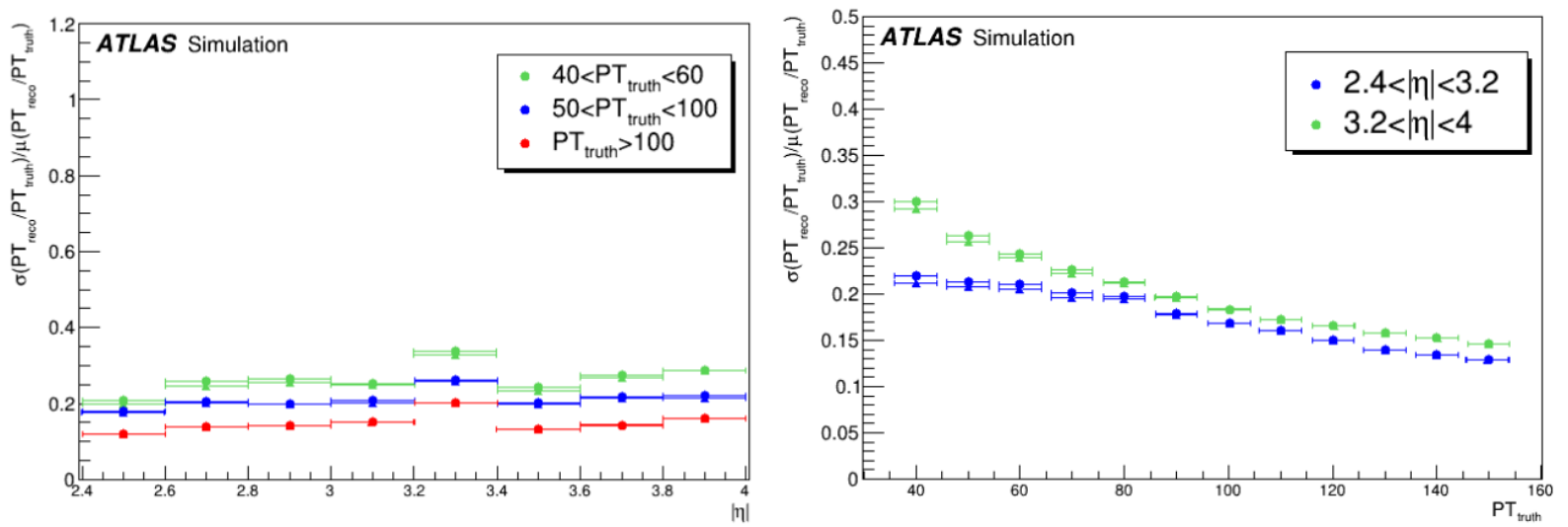

FIGURE 8: The transverse jet energy resolution as a function of $\eta$ (left) and as a function of $p_{\text {Ttruth }}$ (right) before and after correction

\section{CONCLUSION}

The HGTD will provide a timing resolution of 30 ps per minimum-ionizing particle and cover the forward region of the ATLAS detector in $\eta$ between 2.4 and 4.0. This detector is a powerful new tool to address the pileup challenge in the forward region, and can play an important role in many jet/ETmiss HL-LHC studies. The jet performance study shows a promising results for pileup rejection using HGDT.

\section{References}

